\title{
Associations between erythropoietin polymorphisms and risk of diabetic microvascular complications
}

\author{
Hua Li ${ }^{1, *}$, Huipu $\mathrm{Xu}^{2, *}$, Yuerong $\mathrm{Li}^{2}$, Dongdong Zhao ${ }^{2}$ and Baoxin Ma ${ }^{2}$ \\ ${ }^{1}$ Department of Oncology, The Affiliated Hospital of Binzhou Medical University, Binzhou, Shandong 256603, China \\ ${ }^{2}$ Department of Cardiology, The Affiliated Hospital of Binzhou Medical University, Binzhou, Shandong 256603, China \\ "These authors contributed equally to this work
}

Correspondence to: Baoxin Ma, email: sfdbh2006@sohu.com

Keywords: erythropoietin; polymorphism; diabetic microvascular complication; systematic review; meta-analysis Received: May 27, $2017 \quad$ Accepted: October 27, $2017 \quad$ Published: November 27, 2017

Copyright: Li et al. This is an open-access article distributed under the terms of the Creative Commons Attribution License 3.0 (CC BY 3.0), which permits unrestricted use, distribution, and reproduction in any medium, provided the original author and source are credited.

\section{ABSTRACT}

We conducted a meta-analysis to evaluate the relationship between erythropoietin (EPO) polymorphisms and diabetic microvascular complications. We searched the PubMed, Embase, Cochrane library, Web of Science, Wanfang, and Chinese National Knowledge Infrastructure databases for appropriate studies. Odds ratios (ORs) with $95 \%$ confidence intervals (CIs) were calculated to evaluate the associations. Ultimately, eight studies consisting of 2,861 cases and 2,136 controls were identified and included in our meta-analysis. Results with our genotype model indicated an association between rs 1617640 polymorphisms and diabetic microvascular complications (TT vs. GG: $O R=1.544,95 \% C I=1.089-2.189, P=0.015$ ). No clear associations between the rs 1617640 and rs507392 polymorphisms and diabetic retinopathy were observed. By contrast, rs551238 polymorphisms were associated with increased diabetic retinopathy risk (allele model: $\mathrm{OR}=0.774,95 \% \mathrm{CI}=0.658$ $0.911, P=0.002$; genotype model: $A C$ vs. $C C$ : $O R=0.598,95 \% \mathrm{CI}=0.402-0.890, P$ $=0.011$; dominant model: $\mathrm{OR}=0.561,95 \% \mathrm{CI}=0.385-0.817, P=0.003$; recessive model: $O R=0.791,95 \% C I=0.643-0.973, P=0.026)$. These results indicate that EPO polymorphisms are a risk factor for diabetic microvascular complications.

\section{INTRODUCTION}

Diabetes prevalence has increased globally from $4.7 \%$ in 1980 to $8.5 \%$ in 2014 among adults over 18 years of age [1]. Diabetes can damage blood vessels, eyes, and kidneys, resulting in microvascular complications [1-3], and is a contributing factor in $2.6 \%$ of global blindness cases $[1,4]$.

Erythropoietin (EPO) is a kidney-derived peptide hormone that plays a major role in the stimulation of bone marrow stem cells and erythropoiesis [5-7]. EPO can promote retinal angiogenesis independently of VEGF in proliferative diabetic retinopathy $[8,9]$. In diabetic retinas, EPO protects retinal cells by upregulating ZnT8 via ERK pathway activation and HIF-1 $\alpha$ expression inhibition [10], and EPO may slow progression of diabetic nephropathy (DN) [11, 12]. EPO can also improve cardiac function by suppressing endoplasmic reticulum stress and inducing SERCA2a expression [13].
Several studies have examined associations between EPO polymorphisms and diabetic complications; however, the results have been inconsistent. Fan, et al. suggested that erythropoietin polymorphisms increased diabetic retinopathy risk [14]. Conversely, Balasubbu, et al. found no significant association [15]. Thus, our study investigated relationships between single-nucleotide polymorphisms (SNPs) in the EPO gene and diabetic complications.

\section{RESULTS}

\section{Study characteristics}

Figure 1 shows our study selection process. In total, 688 studies were retrieved from the PubMed, Embase, Cochrane library, Web of Science, Wanfang, and Chinese National Knowledge Infrastructure databases. Of these, 151 duplicates were excluded from this study. Another 
493 studies were excluded after reviewing the titles and abstracts. 44 eligible studies were evaluated for full-text review. Of these, 35 were excluded due to non-reporting of available data, duplicate data, or because they were metaanalyses and case reports. Nine original articles containing 12 studies remained after full-text review (Tables 1-2) [14-22].

Hardy-Weinberg equilibrium (HWE) was assessed via Chi-square test in the control subjects of each study. Genotype distributions among controls were consistent with HWE in only eight studies [15-20]. These eight studies, which investigated three SNPs (rs1617640, rs507392 and rs551238), were included in the final metaanalysis. The eight studies included 2,861 cases and 2,136 controls. Five studies focused on diabetic retinopathy, and three focused on diabetic retinopathy and end-stage renal disease. Four examined patients with type 2 diabetes mellitus (T2DM), and one examined patients with type 1 diabetes mellitus (T1DM). Four included patients of Asian descent, three included patients of American descent, and one included patients of Australian descent. According to the Newcastle-Ottawa quality assessment scale (NOS), quality scores ranged from 6-7, and the median score of the case-control studies was 6.5 .

\section{EPO rs1617640 quantitative analyses}

Eight studies investigated the association between EPO rs1617640 gene polymorphisms and diabetic complications risk [15-20]. Differences were observed in the genotype model (TT vs. GG: OR $=1.544,95 \% \mathrm{CI}=$ 1.089-2.189, $P=0.015$ ) (Figure 2, Table 3). For diabetic retinopathy, rs 1617640 polymorphisms were not associated with increased complication risk in any genetic model (allele model: $\mathrm{OR}=0.960,95 \% \mathrm{CI}=0.769-1.198, P=$ 0.717; genotype model: TG vs. GG: $\mathrm{OR}=0.975,95 \% \mathrm{CI}=$ $0.756-1.259, P=0.847, \mathrm{TT}$ vs. $\mathrm{GG}: \mathrm{OR}=1.003,95 \% \mathrm{CI}$ $=0.714-1.410, P=0.985$; dominant model: $\mathrm{OR}=0.988$, 95\% CI $=0.774-1.261, P=0.923$; recessive model: OR $=0.990,95 \% \mathrm{CI}=0.820-1.196, P=0.921)$ (Figure 3). In T2DM and Asian populations, no association was observed in any genetic model (allele model: $\mathrm{OR}=0.940,95 \% \mathrm{CI}=$ $0.721-1.227, P=0.651$; genotype model: TG vs. GG: $\mathrm{OR}=$ $0.983,95 \% \mathrm{CI}=0.753-1.283, P=0.898, \mathrm{TT}$ vs. $\mathrm{GG}: \mathrm{OR}=$ $1.075,95 \% \mathrm{CI}=0.552-2.093, P=0.832$; dominant model: $\mathrm{OR}=1.057,95 \% \mathrm{CI}=0.601-1.859, P=0.846$; recessive model: $\mathrm{OR}=0.980,95 \% \mathrm{CI}=0.804-1.195, P=0.844)$. For diabetic retinopathy and end-stage renal disease, rs 1617640 polymorphisms were associated with diabetic microvascular

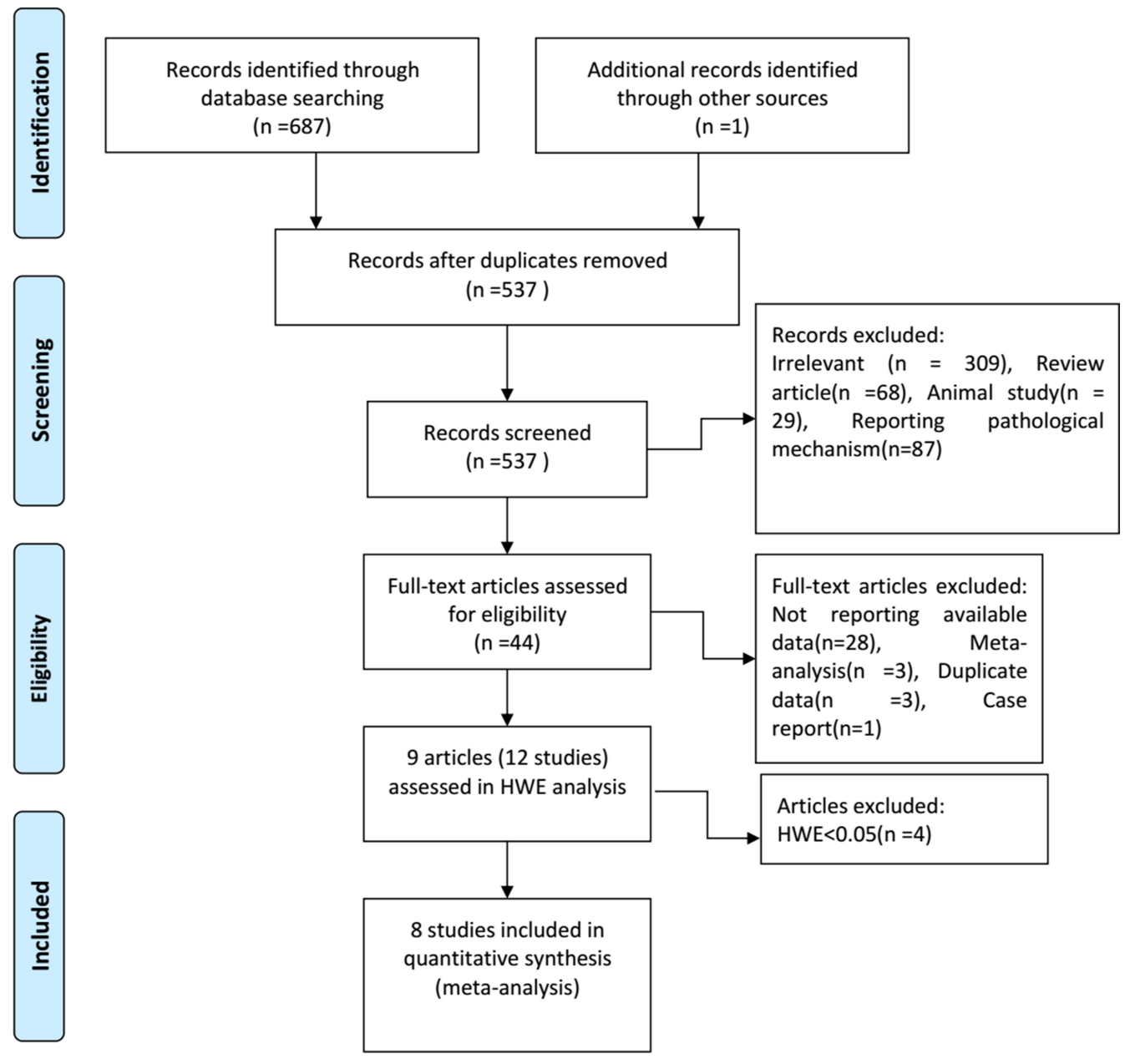

Figure 1: Study selection flow chart. 
Table 1: Main characteristics of studies included in the meta-analysis, by EPO SNP

\begin{tabular}{|c|c|c|c|c|c|c|c|c|c|c|c|c|c|c|c|c|c|c|c|c|}
\hline \multirow[t]{2}{*}{ SNP } & \multirow{2}{*}{$\begin{array}{l}\text { Diabetic } \\
\text { microvascular } \\
\text { complications }\end{array}$} & \multirow[t]{2}{*}{ Author } & \multirow[t]{2}{*}{ Year } & \multirow[t]{2}{*}{ Region } & \multirow{2}{*}{$\begin{array}{l}\text { Type of } \\
\text { diabetes } \\
\text { mellitus }\end{array}$} & \multirow{2}{*}{$\begin{array}{l}\text { Genotyping } \\
\text { method }\end{array}$} & \multirow[t]{2}{*}{ Case } & \multirow[t]{2}{*}{$\begin{array}{l}\text { Con } \\
\text { trol }\end{array}$} & \multicolumn{5}{|c|}{ Case } & \multicolumn{5}{|c|}{ Control } & \multirow[t]{2}{*}{ HWE } & \multirow[t]{2}{*}{$\begin{array}{l}\text { Qua } \\
\text { lity }\end{array}$} \\
\hline & & & & & & & & & $\mathrm{TT}$ & TG & GG & $\mathrm{T}$ & G & TT & TG & GG & $\mathrm{T}$ & $\mathrm{G}$ & & \\
\hline & & $\begin{array}{l}\text { Abhary, } \\
\text { et al. (1) } \\
\text { (T1DM) }\end{array}$ & 2010 & Australia & T1DM & Sequencing & 106 & 67 & 40 & 44 & 18 & 124 & 80 & 24 & 30 & 11 & 78 & 52 & 0.76 & 6 \\
\hline & & $\begin{array}{l}\text { Abhary, } \\
\text { et al. }(2) \\
\text { (T2DM) }\end{array}$ & 2010 & Australia & $\mathrm{T} 2 \mathrm{DM}$ & Sequencing & 179 & 166 & 65 & 78 & 27 & 208 & 132 & 64 & 88 & 11 & 216 & 110 & 0.01 & 6 \\
\hline & Diabetic & $\begin{array}{l}\text { Balasubbu, } \\
\text { et al. }\end{array}$ & 2010 & Indian & $\mathrm{T} 2 \mathrm{DM}$ & $\begin{array}{l}\text { Taqman } \\
\text { assay }\end{array}$ & 345 & 359 & 32 & 163 & 150 & 227 & 463 & 30 & 171 & 158 & 231 & 487 & 0.08 & 6 \\
\hline & retinopathy & Yang, et al. & 2014 & China & $\mathrm{T} 2 \mathrm{DM}$ & Sequencing & 216 & 284 & 146 & 55 & 10 & 347 & 75 & 182 & 82 & 16 & 446 & 114 & 0.11 & 7 \\
\hline & & Zhang & 2014 & China & $\mathrm{T} 2 \mathrm{DM}$ & PCR-LDR & 448 & 344 & 293 & 138 & 13 & 724 & 164 & 225 & 98 & 15 & 548 & 128 & 0.31 & 6 \\
\hline \multirow[t]{9}{*}{ rs 1617640} & & Gong & 2015 & China & $\mathrm{T} 2 \mathrm{DM}$ & $\begin{array}{l}\text { Mass } \\
\text { spectrometry }\end{array}$ & 128 & 128 & 77 & 40 & 11 & 194 & 62 & 92 & 36 & 0 & 220 & 36 & 0.06 & 6 \\
\hline & & $\mathrm{Li}$, et al. & 2016 & China & T2DM & PCR & 191 & 130 & 58 & 121 & 71 & 237 & 263 & 27 & 51 & 9 & 105 & 69 & 0.04 & 7 \\
\hline & & Fan, et al. & 2016 & China & $\mathrm{T} 2 \mathrm{DM}$ & $\begin{array}{l}\text { Taqman } \\
\text { assay }\end{array}$ & 397 & 796 & 208 & 161 & 28 & 577 & 217 & 468 & 302 & 26 & 1238 & 354 & 0.01 & 6 \\
\hline & Diabetic & $\begin{array}{l}\text { Tong, et } \\
\text { al. (1) }\end{array}$ & 2008 & America & $\mathrm{T} 2 \mathrm{DM}$ & SNaPshot & 374 & 239 & 150 & 172 & 52 & 472 & 276 & 66 & 127 & 46 & 259 & 219 & 0.28 & 7 \\
\hline & $\begin{array}{l}\text { retinopathy } \\
\text { and end- } \\
\text { stage renal }\end{array}$ & $\begin{array}{l}\text { Tong, et } \\
\text { al. (2) }\end{array}$ & 2008 & America & T1DM & SNaPshot & 865 & 574 & 335 & 419 & 111 & 1089 & 641 & 148 & 307 & 119 & 603 & 545 & 0.08 & 7 \\
\hline & disease & $\begin{array}{l}\text { Tong, et } \\
\text { al. (3) }\end{array}$ & 2008 & America & T1DM & SNaPshot & 379 & 141 & 139 & 180 & 60 & 458 & 300 & 35 & 78 & 28 & 148 & 134 & 0.20 & 7 \\
\hline & $\begin{array}{l}\text { Diabetic } \\
\text { nephropathy }\end{array}$ & $\begin{array}{l}\text { Alwohhaib, } \\
\text { et al. }\end{array}$ & 2014 & Kuwait & $\mathrm{T} 2 \mathrm{DM}$ & $\begin{array}{l}\text { Taqman } \\
\text { assay }\end{array}$ & 76 & 128 & NA & NA & NA & 73 & 3 & NA & NA & NA & 118 & 10 & NA & 4 \\
\hline & & & & & & & & & TT & $\mathrm{TC}$ & $\mathrm{CC}$ & $\mathrm{T}$ & $\mathrm{C}$ & $\mathrm{TT}$ & $\mathrm{TC}$ & $\mathrm{CC}$ & $\mathrm{T}$ & $\mathrm{C}$ & & \\
\hline & & $\begin{array}{l}\text { Abhary, } \\
\text { et al. (1) } \\
\text { (T1DM) }\end{array}$ & 2010 & Australia & T1DM & Sequencing & 106 & 67 & 40 & 44 & 18 & 124 & 80 & 24 & 30 & 11 & 78 & 52 & 0.76 & 6 \\
\hline \multirow[t]{4}{*}{ rs507392 } & $\begin{array}{l}\text { Diabetic } \\
\text { retinopathy }\end{array}$ & $\begin{array}{l}\text { Abhary, } \\
\text { et al. }(2) \\
\text { (T2DM) }\end{array}$ & 2010 & Australia & $\mathrm{T} 2 \mathrm{DM}$ & Sequencing & 179 & 166 & 65 & 78 & 27 & 208 & 132 & 63 & 88 & 11 & 214 & 110 & 0.01 & 6 \\
\hline & & Zhang & 2014 & China & $\mathrm{T} 2 \mathrm{DM}$ & PCR-LDR & 448 & 344 & 281 & 149 & 14 & 711 & 177 & 225 & 98 & 24 & 548 & 146 & 0.01 & 6 \\
\hline & & Fan, et al. & 2016 & China & $\mathrm{T} 2 \mathrm{DM}$ & $\begin{array}{l}\text { Taqman } \\
\text { assay }\end{array}$ & 397 & 796 & 202 & 161 & 34 & 565 & 229 & 463 & 305 & 28 & 1231 & 361 & 0.01 & 6 \\
\hline & & & & & & & & & $\mathrm{AA}$ & $\mathrm{AC}$ & $\mathrm{CC}$ & A & $\mathrm{C}$ & $\mathrm{AA}$ & $\mathrm{AC}$ & $\mathrm{CC}$ & $\mathrm{A}$ & $\mathrm{C}$ & & \\
\hline \multirow[t]{6}{*}{ rs 551238} & $\begin{array}{l}\text { Diabetic } \\
\text { retinopathy }\end{array}$ & $\begin{array}{l}\text { Abhary, } \\
\text { et al. (1) } \\
\text { (T1DM) }\end{array}$ & 2010 & Australia & T1DM & Sequencing & 106 & 67 & 40 & 44 & 18 & 124 & 80 & 24 & 30 & 11 & 78 & 52 & 0.76 & 6 \\
\hline & & $\begin{array}{l}\text { Abhary, } \\
\text { et al. (2) } \\
\text { (T2DM) }\end{array}$ & 2010 & Australia & $\mathrm{T} 2 \mathrm{DM}$ & Sequencing & 179 & 166 & 65 & 78 & 27 & 208 & 132 & 64 & 88 & 11 & 216 & 110 & 0.01 & 6 \\
\hline & & Zhang & 2014 & China & $\mathrm{T} 2 \mathrm{DM}$ & PCR-LDR & 448 & 344 & 286 & 140 & 13 & 712 & 166 & 219 & 92 & 24 & 530 & 140 & 0.002 & 6 \\
\hline & & Yang, et al. & 2014 & China & $\mathrm{T} 2 \mathrm{DM}$ & Sequencing & 216 & 284 & 141 & 65 & 10 & 347 & 85 & 182 & 79 & 17 & 443 & 113 & 0.04 & 7 \\
\hline & & Gong & 2015 & China & $\mathrm{T} 2 \mathrm{DM}$ & $\begin{array}{l}\text { Mass } \\
\text { spectrometry }\end{array}$ & 128 & 128 & 76 & 40 & 12 & 192 & 64 & 90 & 36 & 2 & 216 & 40 & 0.45 & 6 \\
\hline & & Fan, et al. & 2016 & China & $\mathrm{T} 2 \mathrm{DM}$ & $\begin{array}{l}\text { Taqman } \\
\text { assay }\end{array}$ & 397 & 796 & 203 & 156 & 38 & 562 & 232 & 452 & 299 & 45 & 1203 & 389 & 0.63 & 6 \\
\hline
\end{tabular}

SNP, single-nucleotide polymorphism; PCR-LDR, polymerase chain reaction-ligation detection reaction; T1DM, type 1 diabetes mellitus; T2DM, type 2 diabetes mellitus; NA, not available; HWE, HardyWeinberg equilibrium

complications in all genetic models (allele model: $\mathrm{OR}=$ $1.486,95 \% \mathrm{CI}=1.324-1.667, P=0.000$; genotype model: TG vs. GG: $\mathrm{OR}=1.317,95 \% \mathrm{CI}=1.052-1.648, P=$ 0.016, TT vs. GG: $\mathrm{OR}=2.209,95 \% \mathrm{CI}=1.730-2.821, P$ $=0.000 ;$ dominant model: $\mathrm{OR}=1.607,95 \% \mathrm{CI}=1.298$ $1.990, P=0.000$; recessive model: $\mathrm{OR}=1.792,95 \% \mathrm{CI}=$ $1.502-2.138, P=0.000)$. Only one study assessed T1DM in Australian populations, and no association was found in any genetic model (allele model: $\mathrm{OR}=1.033,95 \% \mathrm{CI}=$ 0.659-1.620, $P=0.886$; genotype model: TG vs. GG: OR $=$ $0.896,95 \% \mathrm{CI}=0.371-2.165, P=0.808$, TT vs. GG: $\mathrm{OR}=$
$1.019,95 \% \mathrm{CI}=0.412-2.517, P=0.968$; dominant model: $\mathrm{OR}=0.951,95 \% \mathrm{CI}=0.417-2.168, P=0.904$; recessive model: $\mathrm{OR}=1.102,95 \% \mathrm{CI}=0.580-2.094, P=0.766)$.

\section{EPO rs507392 quantitative analyses}

Only one study investigated the relationship between EPO rs507392 polymorphisms and diabetic retinopathy [16]. No association was observed in any genetic model (allele model: $\mathrm{OR}=1.033,95 \% \mathrm{CI}=0.659-1.620, P=$ 0.886; genotype model: $\mathrm{TC}$ vs. $\mathrm{CC}: \mathrm{OR}=0.896,95 \% \mathrm{CI}=$ 
Table 2: Main characteristics of studies included in the meta-analysis, by individual study

\begin{tabular}{|c|c|c|c|c|c|c|c|c|c|}
\hline Variables & Abhary (2010) & $\begin{array}{l}\text { Balasubbu } \\
\text { (2010) }\end{array}$ & Yang (2014) & Zhang (2014) & Gong (2015) & Li (2016) & Fan (2016) & Tong (2008) & $\begin{array}{c}\text { Alwohhaib } \\
\text { (2014) }\end{array}$ \\
\hline Ethnicity & Australian & Asian & Asian & Asian & Asian & Asian & Asian & American & Asian \\
\hline $\begin{array}{l}\text { Mean age (SD) } \\
\text { Case vs. Control }\end{array}$ & $\begin{array}{l}\text { T1DM }(P<0.001) \\
48.6(16.0) \text { Vs. } \\
36.3(14.6) \\
\text { T2DM }(P=0.82) \\
64.2(11.1) \text { vs. } 64.5(15.1)\end{array}$ & $\begin{array}{l}57(9) \text { vs. } 59(11) \\
P=0.37\end{array}$ & $\begin{array}{l}50.67(9.41) \text { vs. } \\
52.7(7.61) \\
P=0.008\end{array}$ & $\begin{array}{l}62.35(11.92) \\
\text { vs. } \\
60.16(11.67) \\
P=0.13\end{array}$ & $\begin{array}{l}62.19(8.26) \text { vs. } \\
67.17(9.95) \\
P=0.019\end{array}$ & $\begin{array}{l}63.10(6.83) \\
\text { vs. } \\
68.48(6.91) \\
P<0.001\end{array}$ & $\begin{array}{l}56.1(14.2) \text { vs. } \\
56.9(14.1) \\
P=0.357\end{array}$ & $\begin{array}{l}68 \text { vs. } 70 \\
67 \text { vs. } 44 \\
40 \text { vs. } 52\end{array}$ & NA \\
\hline $\begin{array}{l}\text { Males(\%), } \\
\text { mean(SD) } \\
\text { Case vs. Control }\end{array}$ & $\begin{array}{l}\text { T1DM }(P=0.87) \\
54(51) \text { vs.35(52) } \\
\text { T2DM }(P<0.001) \\
114(63) \text { vs. } 73(44)\end{array}$ & $\begin{array}{l}242(70) \text { vs. } 208 \\
(58) \\
P=0.05\end{array}$ & $\begin{array}{l}103(48) \text { vs. } 111 \\
(39) \\
P=0.054\end{array}$ & $\begin{array}{l}196(44) \text { vs. } \\
163(47) \\
P=0.31\end{array}$ & $\begin{array}{l}61(48) \text { vs. } 58 \\
(45) \\
P=0.707\end{array}$ & $\begin{array}{l}98(51) \text { vs. } 73 \\
(56) \\
P=0.517\end{array}$ & $\begin{array}{l}187(47.1) \text { vs. } \\
392(49.2) \\
P=0.534\end{array}$ & $\begin{array}{l}232(62) \text { vs. } \\
150(63) \\
437(51) \text { vs. } \\
247(49) \\
230(40) \text { vs. } \\
191(50)\end{array}$ & NA \\
\hline $\begin{array}{l}\text { Duration of DM } \\
\text { (years) } \\
\text { mean (SD) } \\
\text { Case vs. Control }\end{array}$ & $\begin{array}{l}\text { T1DM }(P<0.001) \\
28.1(11.7) \text { vs. } 12.43 \\
(8.1) \\
\text { T2DM }(P<0.001) \\
17.3(8.4) \text { vs. } 12.6(8.9)\end{array}$ & $\begin{array}{l}14 \text { (9) Vs. } 14 \text { (9) } \\
P=0.42\end{array}$ & $\begin{array}{l}13.44(7.22) \\
\text { vs. } 14.79(4.97) \\
P=0.013\end{array}$ & $\begin{array}{l}13.61(7.31) \text { vs. } \\
10.70(7.01) \\
P=0.001\end{array}$ & $\begin{array}{l}14.27(6.70) \text { vs. } \\
8.07(5.44) \\
P<0.001\end{array}$ & $\begin{array}{l}15.74(7.48) \\
\text { vs. } 17.74 \\
(7.10) \\
P<0.001\end{array}$ & $\begin{array}{l}9.5(3.8) \text { vs. } \\
5.7(3.0) \\
P<0.001\end{array}$ & $\begin{array}{l}22 \text { vs. } 19 \\
49 \text { vs. } 32 \\
25 \text { vs. } 35\end{array}$ & NA \\
\hline $\begin{array}{l}\text { Smoker (\%), } \\
\text { mean (SD) } \\
\text { Case vs. Control }\end{array}$ & $\begin{array}{l}\text { T1DM }(P=0.93) \\
54(51) \text { vs. } 34(51) \\
\text { T2DM }(P=0.52) \\
99(55) \text { vs. } 86(52)\end{array}$ & NA & NA & NA & NA & NA & $\begin{array}{l}148(37.3) \text { vs. } \\
273(34.2) \\
P=0.321\end{array}$ & NA & NA \\
\hline $\begin{array}{l}\text { HbAlc level, \% } \\
\text { mean (SD) } \\
\text { Case vs. Control }\end{array}$ & $\begin{array}{l}\text { T1DM }(P=0.07) \\
9.2(7.5) \text { vs. } 7.5(2.0) \\
\text { T2DM }(P=0.005) \\
8.5(8.6) \text { vs. } 6.5(3.1)\end{array}$ & $\begin{array}{l}6.4(1.6) \text { vs. } 6.4 \\
(1.2) \\
P=0.09\end{array}$ & $\begin{array}{l}7.84(1.70) \\
\text { vs.6.97 (1.40) } \\
P<0.001\end{array}$ & $\begin{array}{l}9.31(2.88) \text { vs. } \\
8.57(2.03) \\
P=0.07\end{array}$ & $\begin{array}{l}8.06(1.54) \text { vs. } \\
7.74(1.91) \\
P=0.426\end{array}$ & $\begin{array}{l}7.45(0.49) \text { vs. } \\
6.84(0.85) \\
P<0.001\end{array}$ & NA & $\begin{array}{l}8 \text { vs. } 7.6 \\
10.9 \text { vs. } 7.8 \\
7.4 \text { vs. } 8.1\end{array}$ & NA \\
\hline $\begin{array}{l}\text { BMI, mean (SD) } \\
\text { Case vs. Control }\end{array}$ & $\begin{array}{l}\text { T1DM }(P=0.73) \\
25.4(10.1) \text { vs. } 25.9(6.7) \\
\text { T2DM }(P=0.01) \\
29.7(10.9) \text { vs. } 32.5(9.1)\end{array}$ & NA & $\begin{array}{l}25.8(4.13) \text { vs. } \\
25.26(3.95) \\
P=0.14\end{array}$ & $\begin{array}{l}25.58(4.18) \text { vs. } \\
26.16(4.75) \\
P=0.30\end{array}$ & $\begin{array}{l}23.84(2.71) \text { vs. } \\
23.71(2.82) \\
P=0.842\end{array}$ & NA & $\begin{array}{l}23.4(6.7) \text { vs. } \\
25.3(6.2) \\
P<0.001\end{array}$ & NA & NA \\
\hline
\end{tabular}

NA, not available; T1DM, type 1 diabetes mellitus; T2DM, type 2 diabetes mellitus; SD, standard deviation.

Study
ID

Figure 2: Forest plots of the association between rs1617640 polymorphisms and diabetic microvascular complication susceptibility. (TT vs. GG). Squares and horizontal lines correspond to the study-specific OR and $95 \%$ CI. The diamond represents the summary OR and $95 \%$ CI. 
Table 3: ORs and 95\% CIs for EPO gene polymorphisms and diabetic microvascular complications using different genetic models

\begin{tabular}{|c|c|c|c|c|c|c|c|c|c|c|}
\hline \multirow{2}{*}{ SNP } & \multirow{2}{*}{ Comparison } & \multirow{2}{*}{ Subgroup } & \multirow{2}{*}{$N$} & \multicolumn{2}{|c|}{ Heterogeneity test } & \multirow{2}{*}{$\begin{array}{c}\mathrm{Z} \text { test } \\
\mathbf{P}_{\mathrm{Z}}\end{array}$} & \multicolumn{2}{|c|}{ Publication bias } & \multicolumn{2}{|c|}{ OR and $95 \%$ CI } \\
\hline & & & & $\mathbf{P}_{\mathrm{H}}$ & $I^{2}(\%)$ & & $\mathbf{P}_{\mathrm{B}}$ & $\mathbf{P}_{\mathrm{E}}$ & Fixed model & Random model \\
\hline \multirow[t]{35}{*}{ rs 1617640} & T vs. G & Overall & 8 & 0.000 & 76.4 & 0.180 & 0.174 & 0.037 & $1.251(1.146,1.365)$ & $1.142(0.940,1.387)$ \\
\hline & & DR & 5 & 0.049 & 58.1 & 0.717 & 0.221 & 0.356 & $0.991(0.867,1.134)$ & $0.960(0.769,1.198)$ \\
\hline & & $\mathrm{T} 2 \mathrm{DM}$ & 4 & 0.023 & 68.5 & 0.651 & & & $0.987(0.857,1.137)$ & $0.940(0.721,1.227)$ \\
\hline & & T1DM & 1 & & & 0.886 & & & $1.033(0.659,1.620)$ & $1.033(0.659,1.620)$ \\
\hline & & Asian & 4 & 0.023 & 68.5 & 0.651 & & & $0.987(0.857,1.137)$ & $0.940(0.721,1.227)$ \\
\hline & & Australian & 1 & & & 0.886 & & & $1.033(0.659,1.620)$ & $1.033(0.659,1.620)$ \\
\hline & & $\mathrm{DR}+\mathrm{DN}$ & 3 & 0.780 & 0 & 0.000 & & & $1.486(1.324,1.667)$ & $1.485(1.324,1.667)$ \\
\hline & TG vs. GG & Overall & 8 & 0.246 & 23.0 & 0.095 & 0.174 & 0.163 & $1.154(0.975,1.366)$ & $1.169(0.944,1.447)$ \\
\hline & & DR & 5 & 0.202 & 32.9 & 0.847 & 0.221 & 0.462 & $0.975(0.756,1.259)$ & $1.028(0.679,1.555)$ \\
\hline & & T2DM & 4 & 0.118 & 48.9 & 0.898 & & & $0.983(0.753,1.283)$ & $1.040(0.597,1.810)$ \\
\hline & & T1DM & 1 & & & 0.808 & & & $0.896(0.371,2.165)$ & $0.896(0.371,2.165)$ \\
\hline & & Asian & 4 & 0.118 & 48.9 & 0.898 & & & $0.983(0.753,1.283)$ & $1.040(0.597,1.810)$ \\
\hline & & Australian & 1 & & & 0.808 & & & $0.896(0.371,2.165)$ & $0.896(0.371,2.165)$ \\
\hline & & $\mathrm{DR}+\mathrm{DN}$ & 3 & 0.544 & 0 & 0.016 & & & $1.317(1.052,1.648)$ & $1.317(1.051,1.649)$ \\
\hline & TT vs. GG & Overall & 8 & 0.022 & 57.2 & 0.015 & 0.019 & 0.002 & $1.677(1.377,2.043)$ & $1.544(1.089,2.189)$ \\
\hline & & $\mathrm{DR}$ & 5 & 0.147 & 41.2 & 0.985 & 0.221 & 0.092 & $1.003(0.714,1.410)$ & $1.098(0.663,1.820)$ \\
\hline & & $\mathrm{T} 2 \mathrm{DM}$ & 4 & 0.078 & 56.0 & 0.832 & & & $1.001(0.693,1.444)$ & $1.075(0.552,2.093)$ \\
\hline & & T1DM & 1 & & & 0.968 & & & $1.019(0.412,2.517)$ & $1.019(0.412,2.517)$ \\
\hline & & Asian & 4 & 0.078 & 56.0 & 0.832 & & & $1.001(0.693,1.444)$ & $1.075(0.552,2.093)$ \\
\hline & & Australian & 1 & & & 0.968 & & & $1.019(0.412,2.517)$ & $1.019(0.412,2.517)$ \\
\hline & & $\mathrm{DR}+\mathrm{DN}$ & 3 & 0.665 & 0 & 0.000 & & & $2.209(1.730,2.821)$ & $2.208(1.729,2.820)$ \\
\hline & $\begin{array}{c}\mathrm{TT}+\mathrm{TG} \text { vs. } \\
\text { GG }\end{array}$ & Overall & 8 & 0.050 & 50.3 & 0.059 & 0.063 & 0.185 & $1.296(1.104,1.522)$ & $1.294(0.991,1.691)$ \\
\hline & & $\mathrm{DR}$ & 5 & 0.162 & 38.9 & 0.923 & 0.462 & 0.463 & $0.988(0.774,1.261)$ & $1.054(0.692,1.606)$ \\
\hline & & T2DM & 4 & 0.090 & 53.9 & 0.846 & & & $0.992(0.768,1.280)$ & $1.057(0.601,1.859)$ \\
\hline & & T1DM & 1 & & & 0.904 & & & $0.951(0.417,2.168)$ & $0.951(0.417,2.168)$ \\
\hline & & Asian & 4 & 0.090 & 53.9 & 0.846 & & & $0.992(0.768,1.280)$ & $1.057(0.601,1.859)$ \\
\hline & & Australian & 1 & & & 0.904 & & & $0.951(0.417,2.168)$ & $0.951(0.417,2.168)$ \\
\hline & & $\mathrm{DR}+\mathrm{DN}$ & 3 & 0.538 & 0 & 0.000 & & & $1.607(1.298,1.990)$ & $1.607(1.297,1.991)$ \\
\hline & $\begin{array}{c}\text { TT vs. } \\
\text { TG+GG }\end{array}$ & Overall & 8 & 0.001 & 72.2 & 0.087 & 0.019 & 0.002 & $1.365(1.201,1.551)$ & $1.257(0.968,1.632)$ \\
\hline & & $\mathrm{DR}$ & 5 & 0.274 & 22.1 & 0.921 & 0.221 & 0.092 & $0.990(0.820,1.196)$ & $0.986(0.789,1.233)$ \\
\hline & & T2DM & 4 & 0.170 & 40.2 & 0.844 & & & $0.980(0.804,1.195)$ & $0.968(0.740,1.266)$ \\
\hline & & T1DM & 1 & & & 0.766 & & & $1.102(0.580,2.094)$ & $1.102(0.580,2.094)$ \\
\hline & & Asian & 4 & 0.170 & 40.2 & 0.844 & & & $0.980(0.804,1.195)$ & $0.968(0.740,1.266)$ \\
\hline & & Australian & 1 & & & 0.766 & & & $1.102(0.580,2.094)$ & $1.102(0.580,2.094)$ \\
\hline & & $\mathrm{DR}+\mathrm{DN}$ & 3 & 0.981 & 0 & 0.000 & & & $1.792(1.502,2.138)$ & $1.792(1.502,2.138)$ \\
\hline \multirow[t]{5}{*}{ rs507392 } & T vs. C & Overall & 1 & & & 0.886 & & & $1.033(0.659,1.620)$ & $1.033(0.659,1.620)$ \\
\hline & TC vs. CC & Overall & 1 & & & 0.808 & & & $0.896(0.371,2.165)$ & $0.896(0.371,2.165)$ \\
\hline & TT vs. CC & Overall & 1 & & & 0.968 & & & $1.019(0.412,2.517)$ & $1.019(0.412,2.517)$ \\
\hline & $\begin{array}{c}\mathrm{TT}+\mathrm{TC} \text { vs. } \\
\mathrm{CC}\end{array}$ & Overall & 1 & & & 0.904 & & & $0.951(0.417,2.168)$ & $0.951(0.417,2.168)$ \\
\hline & $\begin{array}{l}\text { TT vs. } \\
\text { TC+CC }\end{array}$ & Overall & 1 & & & 0.766 & & & $1.102(0.580,2.094)$ & $1.102(0.580,2.094)$ \\
\hline \multirow[t]{5}{*}{ rs551238 } & A vs. C & Overall & 3 & 0.151 & 47.1 & 0.002 & & & $0.774(0.658,0.911)$ & $0.769(0.585,1.012)$ \\
\hline & $\mathrm{AC}$ vs. $\mathrm{CC}$ & Overall & 3 & 0.225 & 33.0 & 0.011 & & & $0.598(0.402,0.890)$ & $0.596(0.331,1.073)$ \\
\hline & AA vs. CC & Overall & 3 & 0.086 & 59.2 & 0.104 & & & $0.529(0.358,0.782)$ & $0.515(0.231,1.147)$ \\
\hline & $\begin{array}{c}\mathrm{AA}+\mathrm{AC} \text { vs. } \\
\mathrm{CC}\end{array}$ & Overall & 3 & 0.112 & 54.3 & 0.003 & & & $0.561(0.385,0.817)$ & $0.546(0.268,1.111)$ \\
\hline & $\begin{array}{l}\text { AA vs. } \\
\mathrm{AC}+\mathrm{CC}\end{array}$ & Overall & 3 & 0.384 & 0 & 0.026 & & & $0.791(0.643,0.973)$ & $0.791(0.643,0.973)$ \\
\hline
\end{tabular}

$P_{\mathrm{H}}:$ P-value of heterogeneity test; $P_{\mathrm{Z}}:$ P-value of $\mathrm{Z}$ test; $P_{\mathrm{B}}:$ P-value of Begg's test; $P_{\mathrm{E}}:$ P-value of Egger's test; OR: odds ratio; $95 \%$ CI: $95 \%$ confidence interval; N: number of comparisons 
$0.371-2.165, P=0.808$, TT vs. CC: OR $=1.019,95 \%$ CI $=0.412-2.517, P=0.968$; dominant model: $\mathrm{OR}=0.951$, 95\% CI $=0.417-2.168, P=0.904$; recessive model: $\mathrm{OR}=$ $1.102,95 \% \mathrm{CI}=0.580-2.094, P=0.766$.

\section{EPO rs551238 quantitative analyses}

Three studies were eligible for the EPO rs551238 meta-analysis [14, 16, 20]. An association was found between EPO rs551238 polymorphisms and diabetic retinopathy (allele model: $\mathrm{OR}=0.774,95 \% \mathrm{CI}=0.658$ 0.911, $P=0.002$; genotype model: $\mathrm{AC}$ vs. $\mathrm{CC}$ : $\mathrm{OR}=$ $0.598,95 \% \mathrm{CI}=0.402-0.890, P=0.011$; dominant model: $\mathrm{OR}=0.561,95 \% \mathrm{CI}=0.385-0.817, P=0.003$; recessive model: $\mathrm{OR}=0.791,95 \% \mathrm{CI}=0.643-0.973, P=0.026)$ (Figure 4).

\section{Publication bias and sensitivity analysis}

Begg's funnel plot and Egger's test were employed to detect publication bias in this meta-analysis. We found no evidence of publication bias, with the exception of rs1617640 (TT vs. GG, TT vs. TG+GG), which exhibited a slight publication bias (Figure 5, Table 3). A sensitivity analysis was performed to examine the influence of excluding each study on the pooled ORs. The overall effects were not altered when each study was omitted, suggesting that our meta-analysis results were reliable (Figure 6).

\section{DISCUSSION}

EPO is located on chromosome 7q21 and encodes a potent angiogenic factor expressed in the retina and kidney [23-25]. Several studies have assessed the impacts of EPO single-nucleotide polymorphisms (SNPs) on diabetic microvascular complications; however, the results have been inconsistent. We performed this meta-analysis to obtain conclusive results regarding the relationship between EPO polymorphisms and diabetic microvascular complications. We evaluated the most commonly investigated EPO polymorphisms: rs1617640, rs507392, and rs551238. Our results indicate that EPO polymorphisms are a risk factor for diabetic microvascular complications.

We searched several databases for relevant studies assessing the association between the EPO polymorphisms and diabetic complications. Our analysis included eight studies with a combined total of 2,861 cases and 2,136 controls. These studies were conducted between 2008 and 2015 to investigate the association between EPO polymorphisms and diabetic retinopathy and nephropathy. Tong, et al. suggested an association between the T allele of SNP rs1617640 and proliferative diabetic retinopathy as well as end-stage renal disease [19]. Gong associated higher diabetic retinopathy risk with carrying the TT/GT genotype and T allele at the EPO gene site rs1617640 [20]. Abhary, et al. concluded that all three EPO SNPs (rs507392, rs1617640, and rs551238) were associated

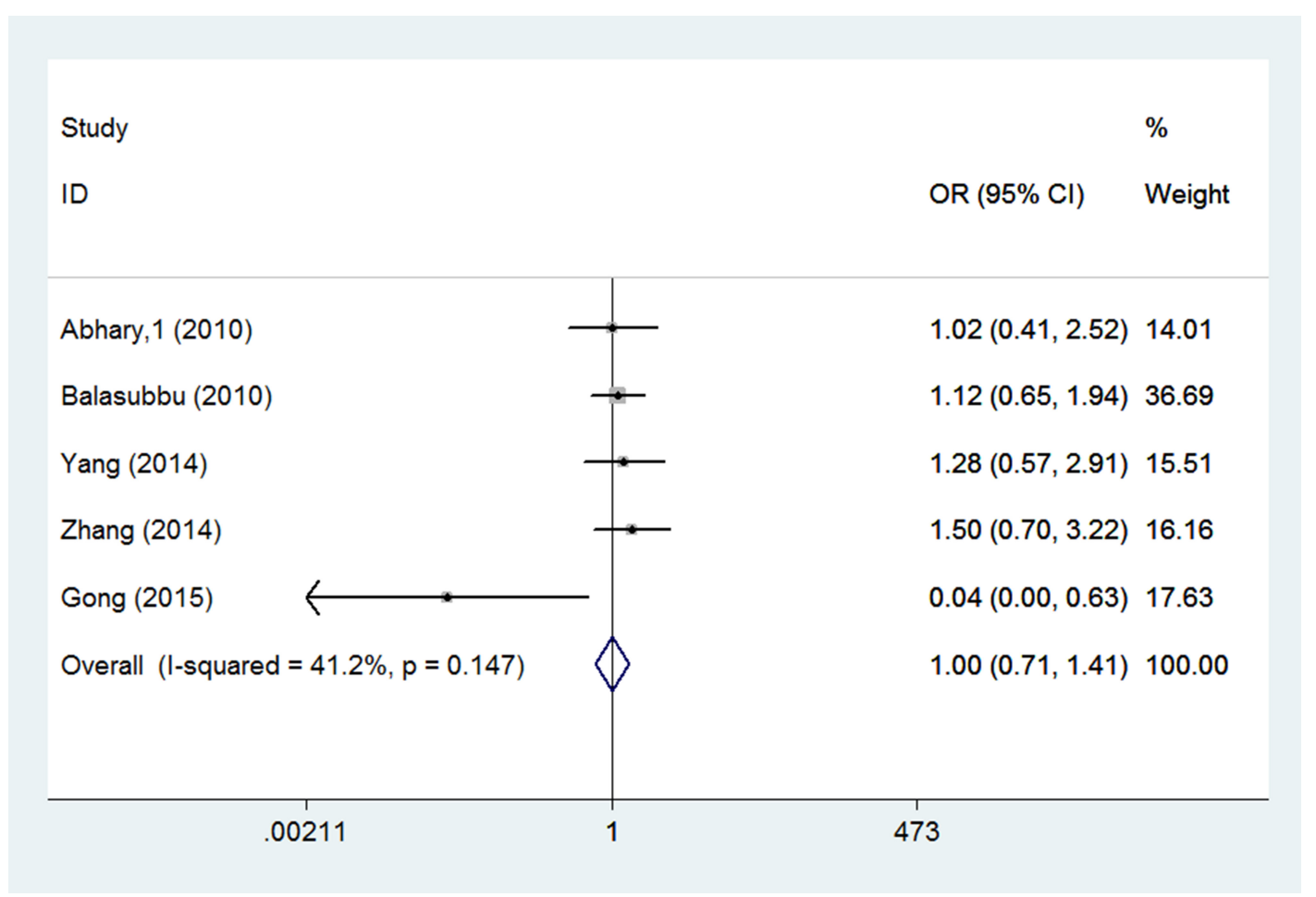

Figure 3: Forest plots of the association between rs1617640 polymorphisms and diabetic retinopathy susceptibility (TT vs. GG). Squares and horizontal lines correspond to the study-specific OR and $95 \%$ CI. The diamond represents the summary OR and $95 \% \mathrm{CI}$. 
with diabetic retinopathy risk independent of duration of DM, degree of glycemic control, and nephropathy [16]. However, some studies found no associations [15]. In our study, the pooled results indicated a relationship between rs1617640 polymorphisms and diabetic complications (genotype model: TT vs. GG: $\mathrm{OR}=1.544,95 \% \mathrm{CI}=$ 1.089-2.189, $P=0.015$ ).

Our subgroup study stratified by complication type showed that rs1617640 polymorphisms were associated with diabetic retinopathy and end-stage renal disease. However, the association with diabetic retinopathy was not significant in any model. Only one study evaluated T1DM in Australian populations, so we were not able to evaluate statistical significance in this case.

Only one study investigated rs507392 polymorphisms, and found no association between these polymorphisms and diabetic retinopathy. Three studies investigated the association between EPO rs551238 polymorphisms and diabetic retinopathy risk, with inconsistent results. We pooled the results and found a relationship between rs551238 polymorphisms and diabetic retinopathy.

Our meta-analysis had some limitations. First, the number of included studies was relatively small. rs1617640 polymorphisms were examined in eight studies, and rs507392 and rs551238 polymorphisms were investigated in one and three studies, respectively. Previous studies demonstrated that EPO variations played roles in DR and DN development [19, 26], and different results were found for T2DM and T1DM. Thus, we performed a subgroup study stratified by complication type, DM type, and ethnicity. However, some of our stratified analyses were limited by small sample sizes. Three studies focused on DR and DN. Only one study assessed T1DM in an Australian population. There were some differences between cases and controls, including patient age, gender, duration of $\mathrm{DM}, \mathrm{HbAlc}$ level and BMI (Table 2). Stratification analyses based on differences in DM duration, gender, or other factors could not be performed because of limited sample sizes. Data from large, multi-center studies, including DM severity, ethnicity, and other complications and factors, are needed to confirm these relationships. Second, due to the limited number of studies, we did not investigate publication bias for the rs551238 polymorphism. A small publication bias was detected for the rs 1617640 polymorphism in some models (TT vs. GG, TT vs. TG + GG), indicating that some unpublished studies might have been overlooked. Third, heterogeneity was found between the rs 1617640 polymorphism studies; however, we could not conduct subgroup analyses to investigate the heterogeneity source due to the limited number of studies. Finally, we did not examine other risk factors, such as environmental effects and genetic factors.

In summary, we found that EPO polymorphisms are a risk factor for diabetic microvascular complications. rs1617640 polymorphisms are associated with increased risk of diabetic retinopathy and nephropathy, and rs551238 polymorphisms are associated with increased risk of diabetic retinopathy.

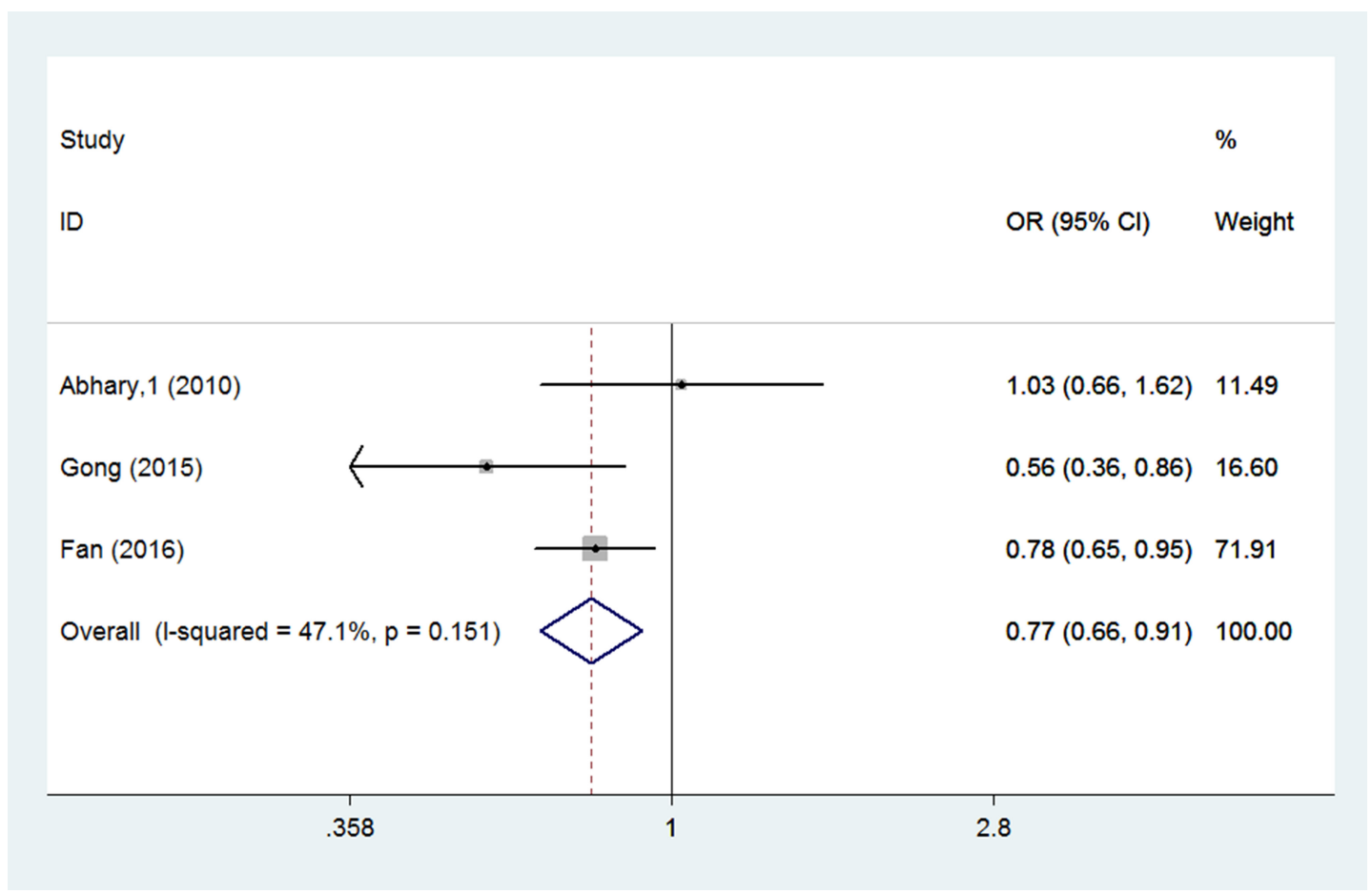

Figure 4: Forest plots of the association between rs551238 polymorphisms and diabetic retinopathy susceptibility (A vs. C). Squares and horizontal lines correspond to the study-specific OR and $95 \%$ CI. The diamond represents the summary OR and $95 \%$ CI. 


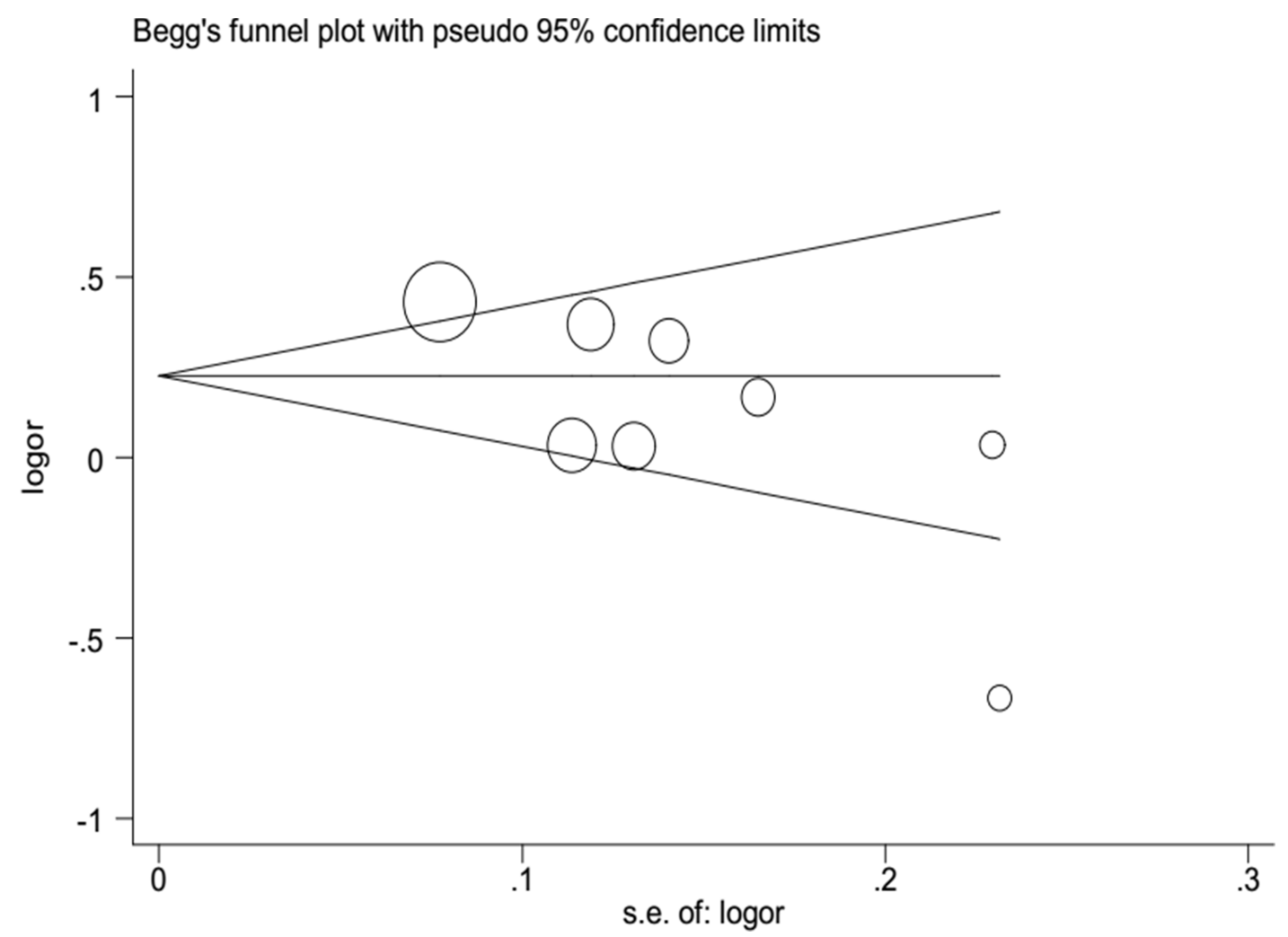

Figure 5: Publication bias tested by Begg's funnel plot (rs1617640 T vs. G). Each point represents a separate study for the indicated association. Logor, natural logarithm of OR. Horizontal line, mean effect size.

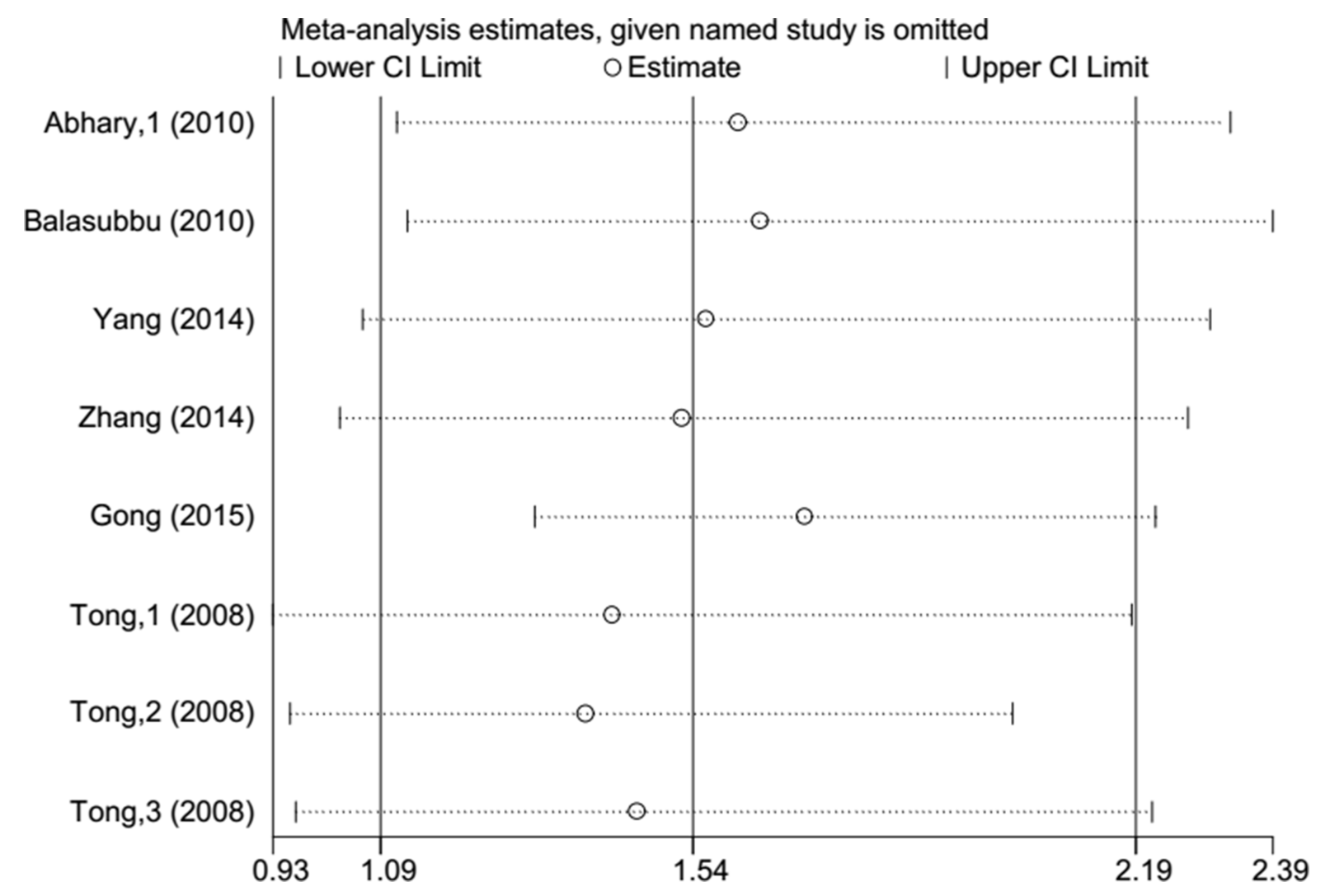

Figure 6: Sensitivity analysis of each study performed by omitting each data set from the analysis (rs1617640 TT vs. GG). 


\section{MATERIALS AND METHODS}

\section{Search for eligible studies}

Relevant studies were retrieved from the PubMed, Embase, Cochrane library, Web of Science, Wanfang, and Chinese National Knowledge Infrastructure databases. The last search was performed on August 22, 2017, with keywords including ("erythropoietin" OR "erythropoietingenerating factor" OR "renal erythropoietic factor" OR "erythropoitin-generating factor" OR "EPO" OR "rs1617640" OR "rs507392" OR "rs551238") AND ("gene" OR "Polymorphisms, Genetic" OR "Polymorphisms, Genetic" OR "Genetic Polymorphism" OR "Polymorphism (Genetics)" OR "Genetic Polymorphisms" OR "Polymorphism" OR "genetic" OR "allele" OR "variation" OR "variant" OR "mutation") AND ("Diabetes" OR "Diabetes-Related " OR "Diabetic ") AND ("Complication" OR "Complications" OR "Disease, Retinal" OR" Diseases, Retinal " OR "Retinal Disease" OR "retinopathy" OR "nephropathy" OR " Disease, Kidney" OR " Kidney Disease" OR "vascular" OR "Blood Vessel" OR "Vessel, Blood" OR "Vessels, Blood" OR "Neuropathy" OR "Neuropathies" OR "Neuralgia" OR "Neuralgias" OR "Cardiomyopathy" OR "Myocardial Diseases" OR "Disease, Myocardial" OR "Diseases, Myocardial" OR "Myocardial Disease" OR "Myocardiopathies" OR "Myocardiopathy"). We also manually searched the reference lists of relevant reports to identify additional studies.

\section{Inclusion and exclusion criteria}

All selected studies in this analysis met the following criteria: (1) case-control studies; (2) explored the correlation between EPO polymorphisms and diabetic microvascular complications; and (3) contained sufficient data for calculating odds ratios (ORs) and $95 \%$ confidence intervals (95\% CIs). Exclusion criteria were as follows: (1) studies without Hardy-Weinberg equilibrium (HWE) in the control groups; (2) studies not relevant to diabetic complications or lacking a control population; and (3) studies lacking sufficient data for quantitative analyses.

\section{Data extraction and quality assessment}

Two investigators ( $\mathrm{Li} \mathrm{H}$ and Xu HP) independently reviewed all articles and extracted available data from individual studies. Any discrepancies were resolved by discussion between these two investigators. The following information was extracted from each eligible study: (1) first author, (2) year of publication, (3) country of origin and ethnicity of study participants, (5) type of diabetes mellitus and complications, (6) genotyping method, (7) number of cases and controls, (8) polymorphism and genotype distribution, and HWE for controls. We assessed the quality of each eligible study according to the
Newcastle-Ottawa quality assessment scale (NOS) [27]. Quality score ranged from $0-9$.

\section{Statistical analysis}

HWE was assessed via Chi-square test in the control populations of each study. Pooled ORs and corresponding 95\% CIs were calculated to estimate associations between EPO polymorphisms and diabetic microvascular complications. $Z$ test was used to assess the overall effect and $P<0.05$ was considered statistically significant. The strength of the association was determined using the following models: allele model (T vs. G, T vs. C, A vs. C), genotype model (TG vs. GG, TT vs. GG, TC vs. CC, TT vs. CC, AC vs. CC, AA vs. CC), dominant model (TT/TG vs. GG, TT/ $\mathrm{TC}$ vs. $\mathrm{CC}, \mathrm{AA} / \mathrm{AC}$ vs. $\mathrm{CC}$ ), and recessive model (TT vs. TG/GG, TT vs. TC/CC, AA vs. AC/CC). Subgroup analyses were conducted according to ethnicity, type of diabetes mellitus, and complication. We used the $Q$-statistic and $\mathrm{I}^{2}$ statistic to evaluate statistical heterogeneity among studies [27]. $P \geq 0.1$ and $\mathrm{I}^{2}<50 \%$ suggested a lack of heterogeneity among studies. A random-effect model was used through the DerSimonian and Laird method in the presence of heterogeneity $\left(P<0.1\right.$ or $\left.\mathrm{I}^{2}>50 \%\right)$; otherwise, a fixed-effect model based on the Mantel-Haenszel method was employed $\left(P \geq 0.1\right.$ or $\left.\mathrm{I}^{2}<50 \%\right)$. We conducted a sensitivity analysis to assess the stability of the results. Egger's test and Begg's funnel plot were used to evaluate publication bias. Analyses were performed using STATA version 11.0 software (Stata Corporation, College Station, TX, USA).

\section{CONFLICTS OF INTEREST}

The authors declare that they have no conflicts of interest.

\section{GRANT SUPPORT}

This research received no specific grant from any funding agency in the public, commercial, or not for-profit sectors.

\section{REFERENCES}

1. World Health Organization. WHO Diabetes Fact Sheet 312, 2016. Available at: http://www.whoint/ mediacentrefactsheets/fs312/en.

2. Hua R, Li Q, Wong IY, Ning H, Wang H. Choroidal microvascular proliferation secondary to diabetes mellitus. Oncotarget. 2017; 8:2034-2036. https://doi.org/10.18632/oncotarget.14020.

3. Al Rasheed R, Al Adel F. Diabetic retinopathy: Knowledge, awareness and practices of physicians in primary-care centers in Riyadh, Saudi Arabia. Saudi J Ophthalmol. 2017; 31:2-6.

4. Chiefari E, Ventura V, Capula C, Randazzo G, Scorcia V, Fedele M, Arcidiacono B, Nevolo MT, Bilotta FL, 
Vitiello M, Palmieri C, Gulletta E, Fusco A, et al. A polymorphism of HMGA1 protects against proliferative diabetic retinopathy by impairing HMGA1-induced VEGFA expression. Sci Rep. 2016; 6:39429.

5. Kodo K, Sugimoto S, Nakajima H, Mori J, Itoh I, Fukuhara S, Shigehara K, Nishikawa T, Kosaka K, Hosoi H. Erythropoietin (EPO) ameliorates obesity and glucose homeostasis by promoting thermogenesis and endocrine function of classical brown adipose tissue (BAT) in dietinduced obese mice. PLoS One. 2017; 12:e0173661.

6. Lamanuzzi A, Saltarella I, Ferrucci A, Ria R, Ruggieri S, Racanelli V, Rao L, Annese T, Nico B, Vacca A, Ribatti D. Role of erythropoietin in the angiogenic activity of bone marrow endothelial cells of MGUS and multiple myeloma patients. Oncotarget. 2016; 7:14510-14521. https://doi.org/10.18632/oncotarget.7587.

7. Kalantar-Zadeh K. History of Erythropoiesis-Stimulating Agents, the Development of Biosimilars, and the Future of Anemia Treatment in Nephrology. Am J Nephrol. 2017; 45:235-247.

8. Takagi H, Watanabe D, Suzuma K, Kurimoto M, Suzuma I, Ohashi H, Ojima T, Murakami T. Novel role of erythropoietin in proliferative diabetic retinopathy. Diabetes Res Clin Pract. 2007; 77:S62-64.

9. Cancarini A, Costagliola C, Dell'omo R, Romano M, Morescalchi F, Agnifili L, Ruggeri G, Semeraro F. Effect of intravitreal bevacizumab on serum, aqueous, and vitreous humor levels of erythropoietin in patients with proliferative diabetic retinopathy. Minerva Endocrinol. 2014; 39:305-311.

10. Xu G, Kang D, Zhang C, Lou H, Sun C, Yang Q, Lu L, Xu GT, Zhang J, Wang F. Erythropoietin Protects Retinal Cells in Diabetic Rats Through Upregulating ZnT8 via Activating ERK Pathway and Inhibiting HIF-1alpha Expression. Invest Ophthalmol Vis Sci. 2015; 56:8166-8178.

11. Eren Z, Gunal MY, Ari E, Coban J, Cakalagaoglu F, Caglayan B, Beker MC, Akdeniz T, Yanikkaya G, Kilic E, Kantarci G. Pleiotropic and Renoprotective Effects of Erythropoietin Beta on Experimental Diabetic Nephropathy Model. Nephron. 2016; 132:292-300.

12. Nand N, Deshmukh AR, Joshi S, Sachdeva MP, Sakthivel. Role of ACE and IL-1beta Gene Polymorphisms in Erythropoeitin Hyporesponsive Patients with Chronic Kidney Disease with Anemia. J Assoc Physicians India. 2017; 65:32-36.

13. Lu J, Dai QM, Ma GS, Zhu YH, Chen B, Li B, Yao YY. Erythropoietin Attenuates Cardiac Dysfunction in Rats by Inhibiting Endoplasmic Reticulum Stress-Induced Diabetic Cardiomyopathy. Cardiovasc Drugs Ther. 2017; 31:367-379.

14. Fan Y, Fu YY, Chen Z, Hu YY, Shen J. Gene-gene interaction of erythropoietin gene polymorphisms and diabetic retinopathy in Chinese Han. Exp Biol Med (Maywood). 2016; 241:1524-1530.

15. Balasubbu S, Sundaresan P, Rajendran A, Ramasamy K, Govindarajan G, Perumalsamy N, Hejtmancik JF.
Association analysis of nine candidate gene polymorphisms in Indian patients with type 2 diabetic retinopathy. BMC Med Genet. 2010; 11:158.

16. Abhary S, Burdon KP, Casson RJ, Goggin M, Petrovsky NP, Craig JE. Association between erythropoietin gene polymorphisms and diabetic retinopathy. Arch Ophthalmol. 2010; 128:102-106.

17. Zhang Y. Association of EPO and TCF7L2 gene polymorphisms with diabetic retinopathy. Shandong University Thesis for Master Degree. 2014.

18. Yang X, Deng Y, Gu H, Ren X, Li N, Lim A, Snellingen T, Liu X, Wang N, Liu N. Candidate gene association study for diabetic retinopathy in Chinese patients with type 2 diabetes. Mol Vis. 2014; 20:200-214.

19. Tong Z, Yang Z, Patel S, Chen H, Gibbs D, Yang X, Hau VS, Kaminoh Y, Harmon J, Pearson E, Buehler J, Chen Y, $\mathrm{Yu}$ B, et al. Promoter polymorphism of the erythropoietin gene in severe diabetic eye and kidney complications. Proc Natl Acad Sci USA. 2008; 105:6998-7003.

20. Gong X. Association Between Erythropoietin Gene Polymorphism And Type 2 Diabetic Retinopathy. Fujian Medical University Thesis for Master Degree. 2015.

21. Li L, Yi XL, Gu YX, Qian Y, Zheng KK. Association of genetic polymorphism of VEGF, ICAM-1 K469E, EPO and TCF7L2 genes with diabetic retinopathy of type 2 diabetes mellitus in Uyghur population in Xinjiang. Journal of Xinjiang Medical University. 2016; 39:1267-1271.

22. Alwohhaib M, Alwaheeb S, Alyatama N, Dashti AA, Abdelghani A, Hussain N. Single nucleotide polymorphisms at erythropoietin, superoxide dismutase 1, splicing factor, arginine/serin-rich 15 and plasmacytoma variant translocation genes association with diabetic nephropathy. Saudi J Kidney Dis Transpl. 2014; 25:577-581.

23. Singh AK, Szczech L, Tang KL, Barnhart H, Sapp S, Wolfson M, Reddan D. Correction of anemia with epoetin alfa in chronic kidney disease. N Engl J Med. 2006; 355:2085-2098.

24. Khoshnood MM, Zareie M, Amiri A, Zarenezhad A, Zarenezhad E, Esfandiari A. The protective effects of erythropoietin on photoreceptor damage by formaldehyde. Nepal J Ophthalmol. 2016; 8:10-17.

25. Wang S, Zhang C, Li J, Niyazi S, Zheng L, Xu M, Rong R, Yang C, Zhu T. Erythropoietin protects against rhabdomyolysis-induced acute kidney injury by modulating macrophage polarization. Cell Death Dis. 2017; 8:e2725.

26. Moemen LA, Hamid MAA, Khalef NA, Elsergany TM, Khlef A. Association between Erythropoietin Gene Polymorphisms and Diabetic Retinopathy. Journal of Applied Sciences Research. 2013; 9:3032-3036.

27. Stang A. Critical evaluation of the Newcastle-Ottawa scale for the assessment of the quality of nonrandomized studies in meta-analyses. Eur J Epidemiol. 2010; 25:603-605. 